\title{
Electronic Structure and Aspects of Unconventional Superconductivity in $\mathrm{Na}_{x} \mathrm{CoO}_{2} \cdot \mathrm{yH}_{2} \mathrm{O}$
}

\author{
H. Rosner, \\ Max-Planck-Institut für Chemische Physik Fester Stoffe, Dresden, Germany \\ S.-L. Drechsler, G. Fuchs, A. Handstein, A. Wälte, and K.-H. Müller \\ Leibniz-Institut für Festkörper- und Werkstoffforschung Dresden (IFW Dresden), P.O. Box 270116, D-01171 Dresden, Germany
}

Received on 23 May, 2003.

\begin{abstract}
We examine the electronic structure of $\mathrm{Na}_{x} \mathrm{CoO}_{2} \cdot \mathrm{yH}_{2} \mathrm{O}$ within the local density approximation. The parametrization of the band which forms the largest hole-Fermi surface centered at $\Gamma$ shows significant deviations from what is frequently assumed in recent sophisticated theoretical studies. In particular, the commonly used nearest neighbor approaches in the framework of single band pictures are found to be unrealistic. The special role of $\mathrm{H}_{2} \mathrm{O}$ in screening the disorder in the charge reservoir is briefly discussed and compared with the case of $\mathrm{Y}_{1-x} \mathrm{Ca}_{x} \mathrm{Cu}_{3} \mathrm{O}_{6+\delta}$.
\end{abstract}

\section{Introduction}

The recent reports on unexpected superconductivity in $\mathrm{Na}_{0.35} \mathrm{CoO}_{2} \cdot 1.3 \mathrm{H}_{2} \mathrm{O}[1]$ below $5 \mathrm{~K}$ and also on magnetic anomalies in closely related $\mathrm{Na}(\mathrm{K})_{x} \mathrm{CoO}_{2+\delta}$ (see Ref. 2) below $31 \mathrm{~K}$ have strongly renewed the interest in layered transition metal oxides. In particular, at present the symmetry of the order parameter and the relationship to cuprates and/or ruthenates are debated controversially. [3] Our paper is organized as follows. The electronic structure calculated in the LDA (local density approximation) is presented in Sec. II. Furthermore consequences for the superconductivity and current theoretical scenarios are briefly discussed. In particular, we propose also a novel solution of the water puzzle in terms of sreening the disorder in the charge reservoir. In Sec. III susceptibility and structural data for nonhydrated samples are reported.

\section{The LDA electronic structure and current theoretical models}

In order to provide a first insight into the involved orbitals and to make a reasonable starting point for more sophisticated but still realistic models, we have performed full potential bandstructure calculations within the FPLO code [4] for $\mathrm{Na}_{0.35} \mathrm{CoO}_{2} \cdot 1.3 \mathrm{H}_{2} \mathrm{O}$ and hypothetical $\mathrm{Na}_{0.35} \mathrm{CoO}_{2}$ with lattice constants of the reference system $\mathrm{Na}_{0.74} \mathrm{CoO}_{2}$. The virtual crystal method has been used to model the partially occupied $\mathrm{Na}$ sites $[5,6]$. To warrant a sufficient completeness of the basis set, the $\mathrm{H}_{2} \mathrm{O}$ sites have been modeled by empty spheres.
Let us start the discussion of our results with the density of states (DOS) near the Fermi energy (see Fig. 1). Two narrow band complexes with predominant Co $3 d$ character occur: one from $e_{g}$-derived states roughly above $2 \mathrm{eV}$ above $E_{f}$ and a $t_{2 g}$-derived one. The strongest peaks near $2 \mathrm{eV}$ and $-1 \mathrm{eV}$ are remnants from 2D Van Hove singularities. The sizable gap between them justifies a low spin approach for the formal Co sites whose charge is formally ${ }^{+3.65} e$.

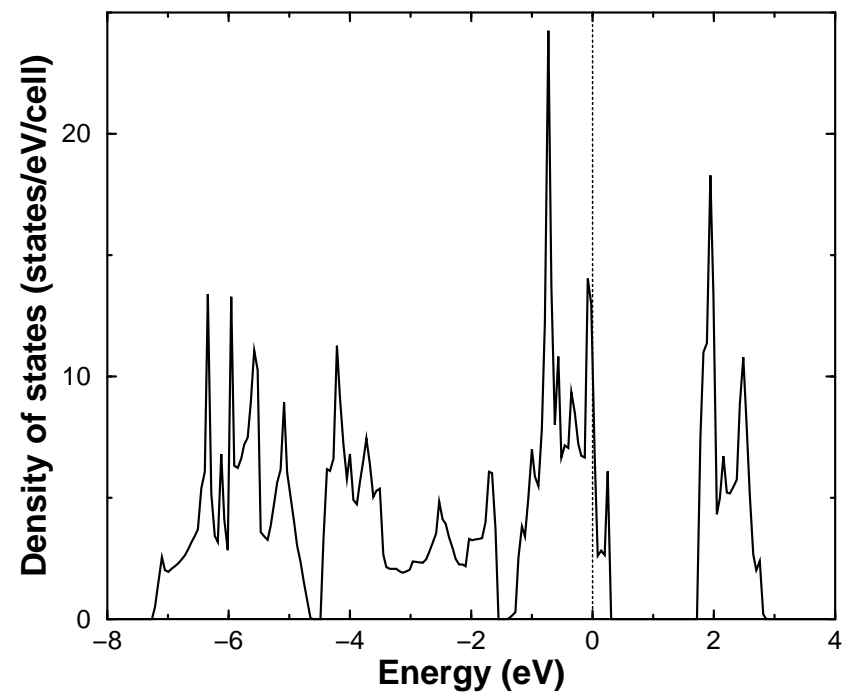

Figure 1. Electronic density of states of $\mathrm{Na}_{0.35} \mathrm{CoO}_{2} \cdot 1.3 \mathrm{H}_{2} \mathrm{O}$. The Fermi energy is at zero energy.

Next we will consider the dispersion of the bands which cross the Fermi energy and the corresponding Fermi surface sheets. The results are qualitatively similar to Ref. 4 for $\mathrm{Na}_{0.5} \mathrm{CoO}_{2}$ although the large lattice expansion in 
c-direction from $11 \AA$ to $19.6 \AA$ due to the presence of $\mathrm{H}_{2} \mathrm{O}$ molecules strongly enhances the two-dimensional (2D) character of the electronic structure (see Figs. 2a,b).
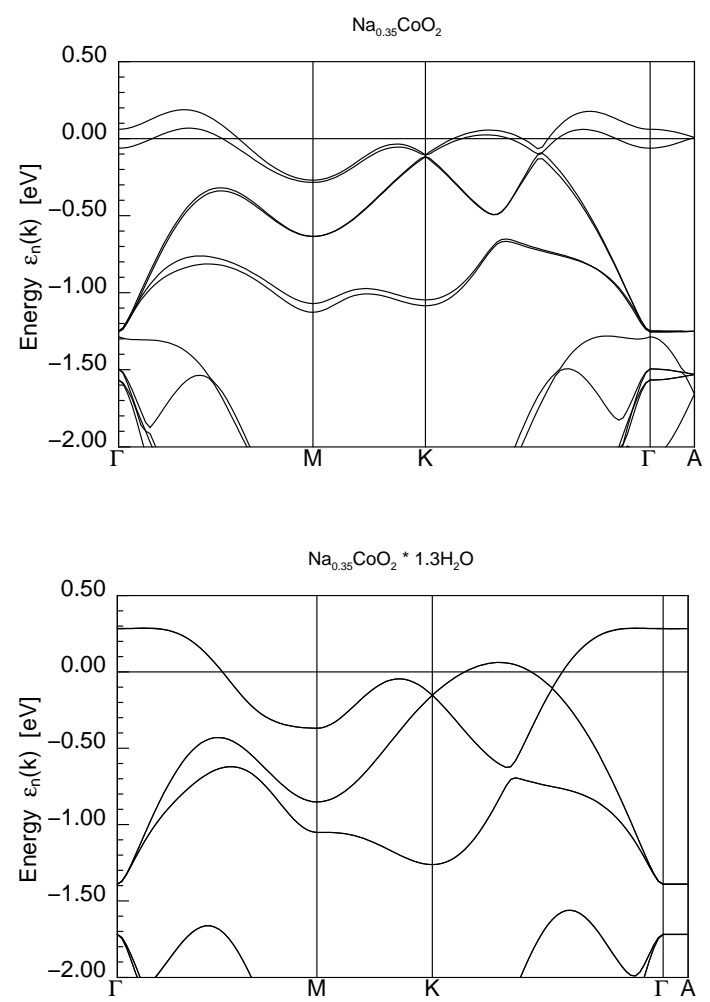

Figure 2. Band structure of (non)hydrous $\mathrm{Na}_{x} \mathrm{CoO}_{2}$, upper and lower panel, resp. The symmetry points read as: $\Gamma=(0,0,0)$; $\mathrm{M}=\frac{2 \pi}{a}\left(\frac{1}{\sqrt{3}}, 0,0\right) ; \mathrm{K}=\frac{2 \pi}{a}\left(\frac{1}{\sqrt{3}}, \frac{1}{3}, 0\right) ;$ and $\mathrm{A}=\frac{2 \pi}{c}(0,0,1)$.

The tiny dispersion along c $4 t_{z} \sim 2 \mathrm{meV}$ causes a huge anisotropy of Fermi velocities $\gamma_{v}=v_{a b} / v_{c} \approx 2 t_{1} a / t_{z} c \approx$ 115. In the Ginzburg-Landau anisotropic mass approximation one would arrive at a huge in-plane/out-of-plane anisotropy for the upper critical fields $H_{c 2}$ of $\gamma_{H} \approx 81$. However, for such extreme anisotropies the latter is usually governed by the intrinsic Josephson-coupling between adjacent layers. The hole Fermi surface (FS) consists of a large sheet centered at $\Gamma$ with a rounded hexagon-like cross section and six small ovoids centered between $\Gamma$ and $\mathrm{K}$ like in $\mathrm{Na}_{0.5} \mathrm{CoO}_{2}$. In our title compound there are also somewhat flattened parts on the large FS. This nesting-like behaviour stems from the interaction of the $t_{2 g}$ derived bands and not from the vicinity of a 2D Van Hove singularity which occurs about $1 \mathrm{eV}$ below $E_{F}$ near the M-point. We have analyzed the bands shown in Fig. 2 within an extended tight-binding (ETB) band including at least also $2^{\text {nd }}$ and $3^{\text {rd }}$ neighbor transfers:

$$
\begin{aligned}
E(\mathbf{k})= & -2 t_{z} \cos z+ \\
& \mu-2 \sum_{n=1}^{3} t_{2 n-1}(\cos 2 n y+2 \cos n x \cos n y)+ \\
& -2 \sum_{n=0}^{1} 2 t_{4 n+2}(\cos 2(n+1) x+ \\
& 2 \cos (n+1) x \cos \sqrt{3} y)+\ldots
\end{aligned}
$$

where $x=\sqrt{3} k_{x} a / 2, y=k_{y} a / 2 . z=k_{z} c / 2$. The result is depicted in Tab. I and Fig. 3,4.

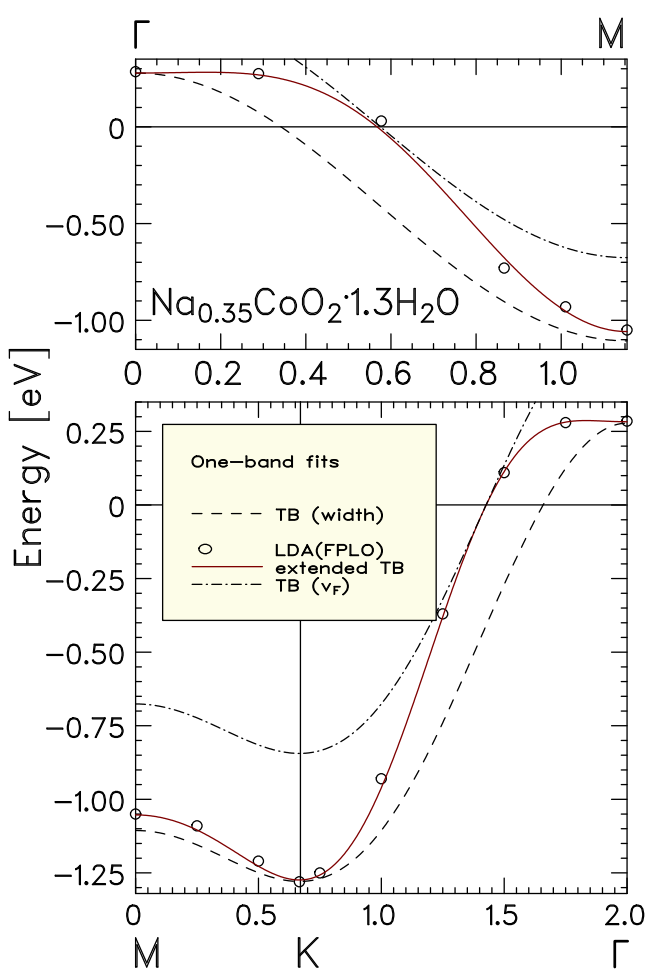

Figure 3. Single band fits for the $a_{1 g}$ band shown in Fig. 2. In the ETB fit $2^{\text {nd }}$ and $3^{\text {rd }}$ neighbors were taken into account while in the simple TB appproximations only either the total band width or the $v_{F}$ /shape of FS (see Fig. 3,4) are properly described. Full line: ETB, dashed line: TB description.

First of all $t_{1}<0$, i.e. $E_{F}$ is far from the Van Hove singularity as mentioned above. Fig. 3 shows that the commonly used TB approximation which enters the corresponding Hubbard or $t$ - $J$-models yields only a poor desription of the whole band which forms the large FS. In order to reproduce the shape of the FS up to $5^{\text {th }}$ neighbors should be taken into account. This reflects the multiband nature of the $t_{2 g}$ band complex and further questions the mentioned single-band approaches. 
Table I. LDA parameters in $\mathrm{meV}$ of $\mathrm{Na}_{0.35} \mathrm{CoO}_{2} \cdot 1.3 \mathrm{H}_{2} \mathrm{O}$ within tight binding (TB)and extended TB (ETB) pictures including $2^{\text {nd }}$ and $3^{\text {rd }}$ neighbor contributions and derived afm Heisenberg exchange integrals compared with literature data.

\begin{tabular}{c|lcccc|ccc}
\hline Reference & $t_{1}$ & $t_{2}$ & $t_{3}$ & $\mu$ & $t_{z}$ & $J_{1, a f m}$ & $J_{2, a f m}$ & $J_{3, a f m}$ \\
\hline ETB & -202 & 35 & 29 & -545 & 0.5 & $40-20$ & $1.2-0.6$ & $0.8-0.4$ \\
TB & -168 & - & - & -340 & 0.5 & $28-14$ & - & - \\
Wang & -250 & - & - & & 0 & & - & - \\
Kumar & +19.4 & - & - & & 0 & 6.5 & - & - \\
Baskaran & -100 & - & - & & 0 & 7 & - & - \\
\hline
\end{tabular}
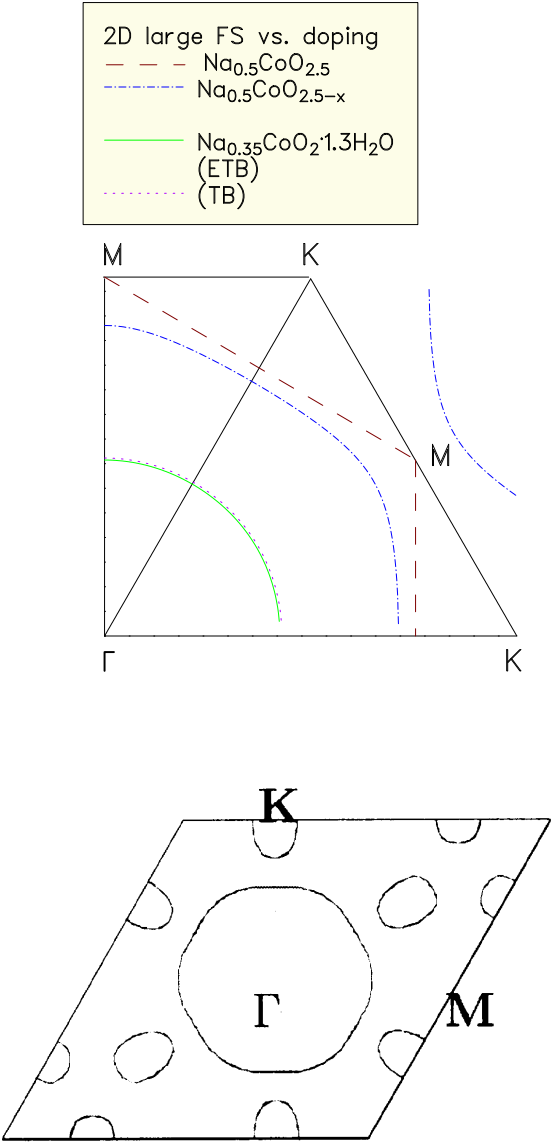

Figure 4. Theoretical Fermi surfaces. Full line: ETB, dashed line: TB description. The dashed-dotted line corresponds to a strongly hole doped case or to the case $t_{1}>0$ not realized in $\mathrm{Na}_{x} \mathrm{CoO}_{2}$. Lower panel: The LDA Fermi surface of the paramagnetic solution.

Another important issue is the magnitude and the sign of the n.n. exchange integral $J_{1}$ :

$$
J_{1}=J_{1, a f m}-J_{1, f m}, \quad \text { where } J_{1, a f m}=4 t_{1}^{2} / U,
$$

With $U=4$ to $8 \mathrm{eV}$ one arrives at $J_{1, a \mathrm{fm}}=40$ to $20 \mathrm{meV}$. Where the first (second) value corresponds to $\mathrm{CoO}_{2}$ regarded as a charge transfer(Mott-Hubbard) insulator, respectively. Even if the ferromagnetic (fm) $J_{1, f m}$ is ignored, a large ratio $\left|t_{1}\right| / J_{1, a f m}=5$ to 10 for the remaining antiferromagnetic (afm) part results, again in contrast with significantly smaller values of 1 to 3 adopted and studied in the literature. More importantly, the very existence of a considerable afm exchange on the frustrated triangular Co lattice which is of crucial importance for the proposed sophisticated resonance valent bond (RVB)-type scenarios [7, 8] is unclear. Since our LSDA calculations [6] yield a fm ground state with an energy gain $160 \mathrm{meV}$ and a magnetic moment of $0.66 \mu_{B} / \mathrm{Co}$, sizeable direct fm contributions to $J_{1}$ are expected. The experimental lack of a ferromagnetically ordered state predicted by the LSDA solution might be attributed to its mean-field character where non-negligible fluctuations important especially for low-dimensional systems are ignored. However, the energy difference to an antiferromagnetic state is relatively small, a more sophisticated account of correlation and fluctuation effects beyond the LSDA maybe crucial for the title compound being near the border line of two different magnetic ground states. Similarly the symmetry of the superconducting order parameter will be strongly affected by the final choice. In addition, the inspection of the Co-O-Co bond angles $\approx 90^{\circ}$ suggests $\mathrm{fm}$ or strongly suppressed afm interactions. In the case of triplet $p$ - or $f$-wave superconductivity which is likely to occur, the remaining intrinsic disorder deserves closer considerations. In this context the sensitive effect of crystal water or of $\mathrm{O}$ excess ions, respectively, might be considered in terms of their high polarizibility, i.e. screening, and larger distances between $\mathrm{Na}$ and $\mathrm{CoO}_{2}$ layers and this way weakening the detrimental effect of disorder from randomly located $2 x \mathrm{Na}^{+}$-ions at the two Na sites per unit cell.

This effect resembles the strange occurence/vanishing of superconductivity in $\mathrm{Y}_{1-x} \mathrm{Ca}_{x} \mathrm{Ba}_{2} \mathrm{Cu}_{3} \mathrm{O}_{6+\delta}$ [9] where despite the presence of nearly the same significant hole concentration in the $\mathrm{CuO}_{2}$ planes (due to Ca doping) as in the ortho-II phase of $\mathrm{YBa}_{2} \mathrm{Cu}_{3} \mathrm{O}_{6.5}\left(T_{c}=60 \mathrm{~K}\right)$, the actual $T_{c}$ depends critically on a small amount of random $\mathrm{O}(\delta \leq 0.1)$ and holes on the apical oxygen. For small $\delta \sim 0.05$ the ordinary "stick" layer built by relatively unpolarizable $\mathrm{Cu}(1) \mathrm{O}_{2}$ rods contains also few highly polarizable ionic $\mathrm{Cu}(1)_{2} \mathrm{O}_{5}$ "biradicals". The presence of the latter causes a critical down screening of the strong fluctuating electrostatic potential at the $\mathrm{CuO}_{2}$ layers. These fluctuations arise from the random 


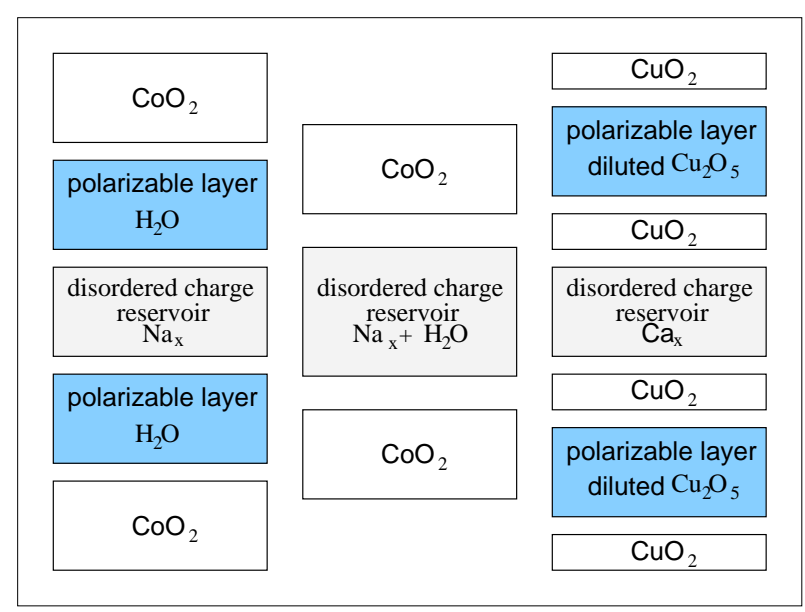

Superconduct. Nonsupercond. Superconduct. Figure 5. Schematical structure of $\mathrm{Na}_{0.35} 1.3 \mathrm{H}_{2} \mathrm{OCoO}_{2}$ (left panel) and $\mathrm{Y}_{1-x} \mathrm{Ca}_{x} \mathrm{Ba}_{2} \mathrm{Cu}_{3} \mathrm{O}_{6+\delta}$ (right panel), two metallic compounds with superconductivity induced by the screening of the detrimental intrinsic disorder in their charge reservoirs by adding polarizable molecules (radicals) outside their conduction layers. The nonsuperconducting compound $\mathrm{Na}_{0.35} 0_{0.6 \mathrm{H}_{2}} \mathrm{OCoO}_{2}$ (middle panel) with almost identical electronic structure of the $\mathrm{CoO}_{2}$ layers but unfavorable positions and a less amount of the polarizable $\mathrm{H}_{2} \mathrm{O}$ molecules ( now located at noneffective sites inside the charge reservoir).

Ca occupation within the $\mathrm{Y}_{1-x} \mathrm{Ca}_{x}$ charge resevoir within the cuprate bilayer. In this context the lack of superconductivity in $\mathrm{Na}_{0.35} \mathrm{CoO}_{2} \cdot 0.6 \mathrm{H}_{2} \mathrm{O}$ [10] with only half as much water content at different position (within the Na-layer) and distance between the $\mathrm{CoO}_{2}$ and the Na layers (3.45 $\AA$ ) compared with the hydrous superconducting compound (4.9 $\AA$ ) is of interest. It corresponds to the nonsuperconducting cuprate counterpart with $\delta=0$ in spite of the sufficient concentration of doped holes in the $\mathrm{CuO}_{2}$ planes. Alternatively, the improved screening might also weaken or suppress a charge ordered ground state as proposed recently by G. Baskaran[11]. The charge ordered state is expected to compete with superconductivity. However, the specific triangular $\mathrm{CoO}_{2}$ block structure causes considerable frustration effects not only for an afm ordering which is at the heart of the afm RVB models discused above but also for any charge ordering.

\section{Magnetic susceptibility measure- ments}

Finally, preliminary sample processing adopted from Ref. 2 yield X-ray patterns similar to $\mathrm{Na}_{0.74} \mathrm{CoO}_{2}$ (see Fig. 6).

Preliminary magnetic susceptibility measurements showed so far no clear hints for superconductivity in the nonhydrous compound but point rather to a magnetic transition near $22 \mathrm{~K}$ (see Fig. 7). Anyhow, at present it cannot be fully excluded, although it is rather unlikely, that the few superconducting samples of Ref. 2 are related to hole doped superconductivity like in $\mathrm{Na}(\mathrm{K})_{2 \delta-0.5} \mathrm{CoO}_{2+\delta}$ near 1/4-filling (count in electrons).

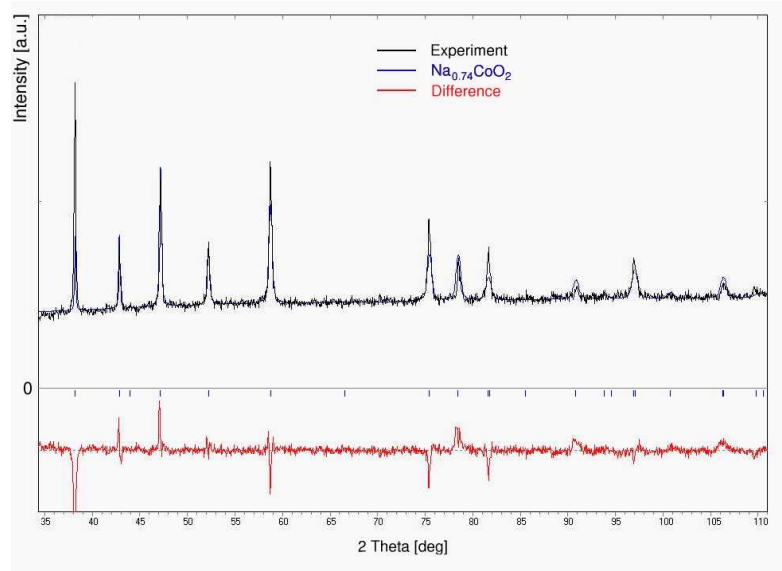

Figure 6. X-ray diffraction pattern of the sample whose magnetic susceptibility is shown in Fig. 7.

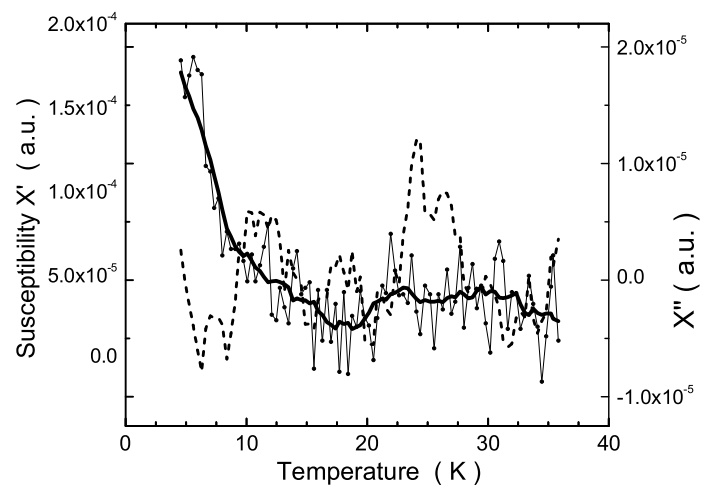

Figure 7. Real (full line) and imaginary (broken line) parts of the magnetic susceptibility (smoothened) of water free $\mathrm{Na}_{x} \mathrm{CoO}_{2}$ prepared as described in Ref. 2.

Anyhow, the study of hole doped cobaltates would be of great interest even in the case of lacking superconductivity.

\section{Conclusion}

To summarize, the parameters of most of the recently proposed single band tight binding approximations entering Hubbard or $t$ - $J$-models are in some conflict with the multiband nature predicted by the LDA. A solution of the water puzzle, the screening of the disorder detrimental for $p$-wave superconductivity, has been proposed.

\section{Acknowledgments}

The DFG under project SPP 1073 and the SFB 463 are gratefully acknowledged for financial support. We thank $\mathrm{H}$. Eschrig, W.E. Pickett, J. Röhler, R. Cava, G. Baskaran, N. Nücker, S. Schuppler, S.V. Shulga and G. Krabbes for useful discussions and stimulating interest. 


\section{References}

[1] K. Takada et al. Nature 422, 63 (2003).

[2] H.-H. Wen, et al., Chin. Phys. Lett. 20, 725 (2003), v. 4 condmat/0304377. Due to the low reproducibility (only 3 unidentified samples from 100 exhibited superconductivity) this paper has been withdrawn by the authors.

[3] The work devoted to this topic is still given by a fastly growing number of preprints on the cond-mat archive.

[4] K. Koepernik and H. Eschrig, Phys. Rev B 59,1743 (1999).

[5] D. Singh, Phys. Rev. B 61, 13397 (2000).

[6] D. Singh, cond-mat/0304532.

[7] G. Baskaran, preprint, cond-mat/0303649.

[8] B. Kumar and S. Shastry, preprint, cond-mat/0304210.

[9] M. Merz et al., Phys. Rev. Lett. 80, 5192 (1998). The authors attribute the superconductivity in the samples with $\delta \sim 0.05$ to an active role of the apical oxygen ions in the pairing. However, due to the small amount of relevant ionic $\mathrm{Cu}_{2} \mathrm{O}_{5}$ biradicals (see also Fig. 5) such an active scenario seems to us rather unlikely and we prefer the indirect effect by screening the intrinsic disorder as proposed above as a more realistic one.

[10] M.L. Foo et al., cond-mat/0304464.

[11] G. Baskaran, cond-mat/0306569, Physica C in press.

\section{Note added in proof}

Three recent ARPES measurements performed for anhydrous $\mathrm{Na}_{x} \mathrm{CoO}_{2}$ samples with $x=0.5,0.6$, and 0.7 ( $\mathrm{T}$. Valla et al., Nature 417, 627 (2002); H.-B. Yang et al., cond-mat/0310532 and references therein) confirm the negative sign value of the main transfer integral $t_{1}$ predicted by the LDA calculations but at the same time show that it is strongly reduced in its absolute value pointing to a strong renormalization. Since a similar large mass renormalization has been suggested by a large electronic specific heat coefficient, strong ferromagnetic spin fluctuations (paramagnons) are a likely candidate as proposed above. Antiferromagnetic spin fluctuations and other correlation effects are expected to cause weaker renormalization effects, especially for the specific heat.

Unfortunately, to the best of our knowledge, there are no data available in the lower doping range $x \sim 0.3$ to 0.4 which is of interest for the superconductivity where antiferromagnetic fluctuations might be enhanced. Quite remarkably, for the three dimensional-s-wave superconductor $\mathrm{MgCNi}_{3}$ (which is also more or less close to a ferromagnetic instability) some similarities with the cobaltates under consideration with respect to the normal state electronic specific heat have been observed (Wälte et al., cond-mat/0208364 and further work to be published).

Our spin polarized LSDA results agree with the findings reported recently by J. Kunecš et al., cond-mat/03082888. 